ARTICLE

\title{
Application of the Multi-Model Monte-Carlo Treatment Planning System Combined with PHITS to Proton Radiotherapy
}

\author{
Hiroaki KUMADA ${ }^{1, *}$, Takeji SAKAE ${ }^{1}$, Kimiaki SAITO$^{2}$, Tomonori ISOBE ${ }^{1}$ \\ Takayuki HASHIMOTO ${ }^{1}$ and Hideyuki SAKURAI ${ }^{1}$ \\ ${ }^{1}$ University of Tsukuba, 1-1-1 Tennodai, Tsukuba-shi, Ibaraki-ken, 305-8575, Japan \\ ${ }^{2}$ Japan Atomic Energy Agency, 2-4 Shirakata, Tokai-mura, Naka-gun, Ibaraki-ken, 319-1195, Japan
}

\begin{abstract}
In proton radiotherapy, a proton generates secondary neutrons and photons in a patient's body. This leads to irradiation of the area in proximity to the target region with incidental doses caused by secondary radiation in addition to the therapeutic dose. However in current treatment planning of proton radiotherapy, conventional dose estimation algorithms like the pencil beam method can not estimate the influence of secondary radiation. Thus, to improve accuracy of radiotherapy's dose estimation, a new multi-modal Monte-Carlo treatment planning system is being developed. The new system employs the Monte-Carlo transport code PHITS as the dose calculation engine of the system. The final goal of this study is to investigate the application of the Monte-Carlo treatment planning system at the proton radiotherapy facility of the University of Tsukuba. To achieve this goal, first, the influence of the secondary radiations and the incidental doses generated by the proton irradiation was estimated by the Monte-Carlo simulation utilizing PHITS. Secondly, we aim at performing Monte-Carlo treatment planning for proton radiotherapy using JCDS-FX combined with PHITS.

The estimation results for the secondary radiation caused by proton irradiation demonstrated that the maximum value of absorbed dose due to recoil protons was approximately $0.64 \mathrm{mGy} / \mathrm{Gy}$ at a depth of $6.5 \mathrm{~cm}$ on the central axis of the phantom. The absorbed dose accounted for about $72 \%$ of the total incidental equivalent dose. A lot of external neutrons generated at upstream devices irradiate the surface of the phantom. However, at deeper located regions, influences of the external neutrons were small as neutrons generated inside the body accounted for a large amount of the total neutron count in this region. The results demonstrated that it is impossible to eliminate the incidental dose completely, even if shielding of the external neutrons and application of the active scanning method are achieved.
\end{abstract}

KEYWORDS: proton radiotherapy, treatment planning, Monte-Carlo, computational dosimetry, BNCT

\section{Introduction}

Proton radiotherapy treating various types of cancer patients is being performed at the Proton Medical Research Center (PMRC) of the University of Tsukuba. ${ }^{1)}$ To determine the therapeutic dose given to a patient, the treatment planning for proton radiotherapy at PMRC uses the pencil beam method as a type of dose calculation algorithm. ${ }^{2,3)}$ At present, the pencil beam method is employed to most treatment planning systems of radiotherapy as the application of this method allows to determine the dose distribution around the irradiation field within a short time. However, the method can not estimate scattered radiation of the proton beam along the beam path adequately. It is also impossible to determine secondary neutrons and photons generated by the reactions of protons with several elements of body tissue, thus influence of incidental dose caused by secondary radiation are not estimated in the treatment planning if this method is used.

At present, we are developing a Monte-Carlo based multi-modal treatment planning system in cooperation with the Japan Atomic Energy Agency (JAEA), ${ }^{4,5)}$ The new system (developing code: JCDS-FX) builds on fundamental tech-

\footnotetext{
*Corresponding author,E-mail:kumada@pmrc.tsukuba.ac.jp
}

(C) 2011 Atomic Energy Society of Japan, All Rights Reserved. nologies of JCDS. JCDS, the forerunner of JCDS-FX is a treatment planning system for boron neutron capture therapy (BNCT), a subtype of particle radiotherapy. JCDS has shown a lot of practical benefits in BNCT clinical trials performed at Japan Research Reactor No.4 (JRR-4) in JAEA. ${ }^{6,7)}$ Figure 1 shows the procedure schema of treatment planning with JCDS-FX.

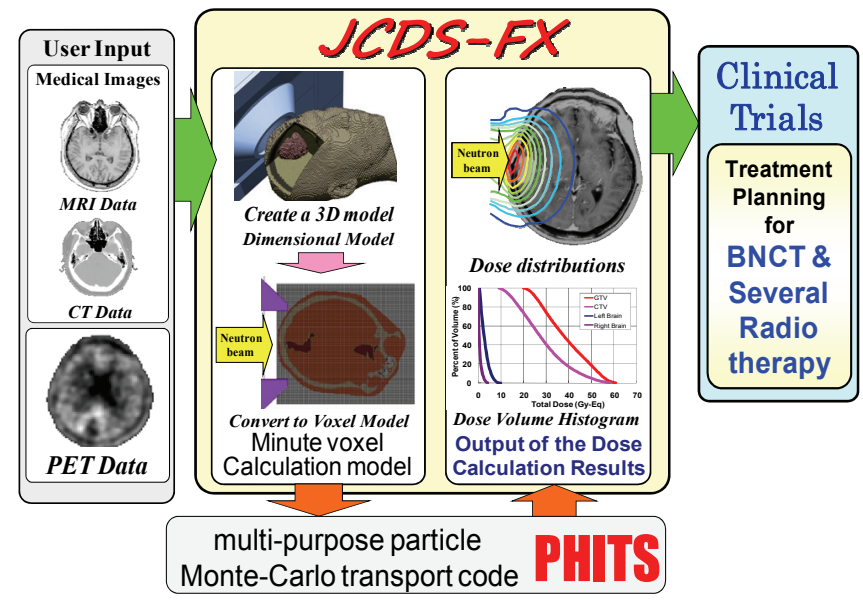

Fig. 1 Treatment planning procedure using JCDS-FX 
JCDS-FX offers more features and possesses several advantages over JCDS. One of the features of JCDS-FX is that PHITS has been applied to dose calculation. PHITS is a multi-purpose particle transport code, the Monte-Carlo code can deal with particle transport for not only neutrons and photons but also protons, heavy ions and electrons. ${ }^{8)}$ Thus, application of PHITS as dose calculation engine of JCDS-FX enabled us to perform treatment planning for charged particle radiotherapy as well as BNCT. Therefore, we intend to apply JCDS-FX combined with PHITS to proton radiotherapy at PMRC in order to improve the dose estimation accuracy of the treatment planning and Quality Assurance/Quality Control of radiotherapy.

The proton irradiation method applied at PMRC is the passive scattering method for which the distribution of the therapeutic dose in the target region is formed by using a ridge filter, range shifter, bolus and collimator. In the radiotherapy, the proton beam generates fast neutrons by reacting with devices located along the beam path. The proton also generates secondary neutrons and photons by the reaction with several elements that form human body tissue. Incidental doses caused by secondary radiation are affecting the area in proximity to the target region in addition to the therapeutic dose. Furthermore, in case of a patient being equipped with a pacemaker or an implantable cardiac defibrillator (ICD), the neutrons might induce functional interference with the device during the irradiation. ${ }^{9,10)}$ However, in proton radiotherapy at present, incidental doses and secondary radiation do not count toward dose limits in treatment planning due to the application of the efficient dose determination system which utilizes the pencil beam method as described above.

The final goal of this study is to apply Monte-Carlo treatment planning with JCDS-FX to proton radiotherapy performed at PMRC of the University of Tsukuba. To achieve this goal, first, we estimate the influence of the secondary radiations and the incidental doses caused by proton irradiation by use of Monte-Carlo simulation. The secondary radiations and incidental doses are determined by PHITS which is employed by JCDS-FX. We simulate the proton irradiation experiment with a water phantom which had been performed in PMRC in order to measure the influence of secondary radiation with regard to pacemakers or ICDs experimentally. In the estimation, several absorbed doses were generated by the reaction of secondary radiation with each element which forms the human body such as hydrogen and nitrogen. Next, we perform Monte-Carlo treatment planning for proton radiotherapy by using JCDS-FX.

\section{Materials and Methods}

\section{Phantom Experiments for Influence Analysis of Sec- ondary Radiation with Pacemakers and ICDs}

To investigate the influence of secondary radiation with pacemakers and ICDs experimentally, phantom experiments were performed at PMRC. An overview of the phantom experiment setup is given in Fig. 2.

The phantom was composed of water with dimensions $24 \times 24 \times 24 \mathrm{~cm}^{3}$ and the entrance of the phantom was located

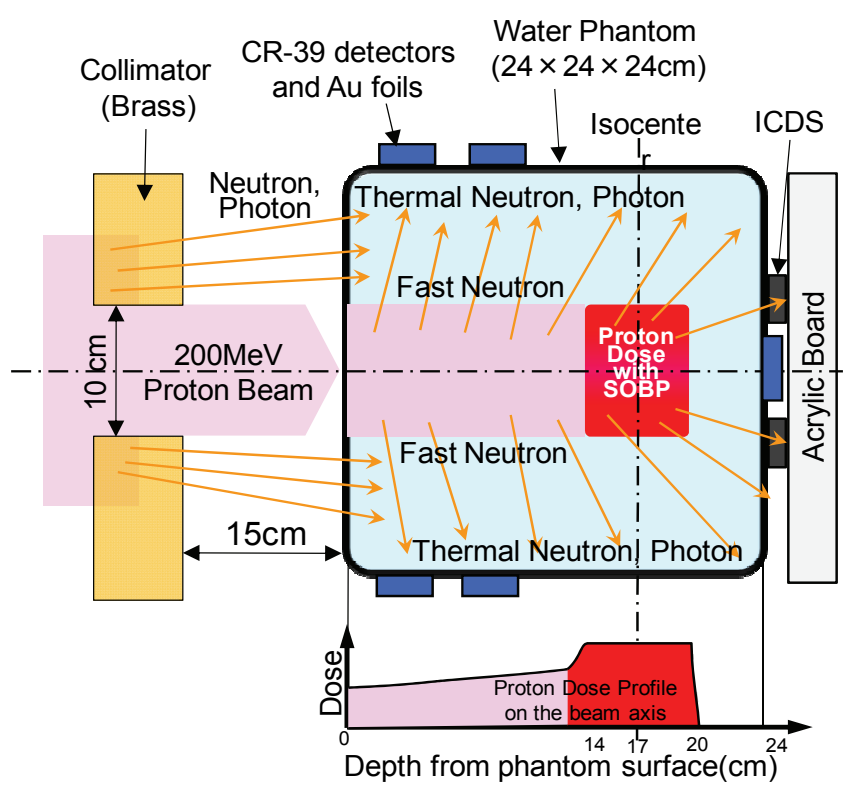

Fig. 2 Schema of the phantom experiment

$15 \mathrm{~cm}$ away from the downstream face of the collimator which itself is installed inside the treatment nozzle. The collimator made of brass acts as a beam aperture resulting in a $10 \times 10 \mathrm{~cm}^{2}$ square shape. Several pacemakers and ICDs were set up behind the phantom. Additionally, gold foils and several CR-39 detectors (Luxel badge; Nagase Landauer, Ibaraki, Japan) acting as neutron and photon detectors were also set out on the surface of the phantom in order to measure the neutron dose, ${ }^{11)}$ and gamma-ray dose at that point of interest as shown in Fig. 2. The activity of the irradiated gold foils was measured by using a germanium detector. ${ }^{12)}$ With the measurement results, the thermal neutron flux could be determined. Uncertainties of the thermal neutron flux measurement were estimated to be $2.9 \%$ (average \pm 1 standard deviation). In the experiments, a $200 \mathrm{MeV}$ proton beam was used to irradiate the center of the phantom, generating a SOBP of $6 \mathrm{~cm}$ in length at a depth of $14 \mathrm{~cm}$ to $20 \mathrm{~cm}$ in the phantom.

\section{Determination of the Secondary Radiation and Inci- dental Doses by Using PHITS}

Clasie et $a l .{ }^{13)}$ published estimations of the incidental doses caused by secondary radiation during proton irradiation by making use of Monte-Carlo simulation. Proton irradiation experiments with a Lucite phantom, which was performed in the facility of Francis H. Burr Proton Therapy Center, were simulated by using GEANT4 as a Monte-Carlo calculation code. ${ }^{14)}$ Finally, they determined distributions of absorbed dose and equivalent dose in the phantom. Correspondingly, we also simulated the phantom experiment performed in the PMRC using PHITS. In the simulation, the geometry of the proton irradiation devices such as ridge filter, range sifter and multi-leaf collimator were configured properly. Figure 3 shows the arrangement of several proton irradiation devices implemented at PMRC. The water phantom model was positioned at the irradiation point in the 


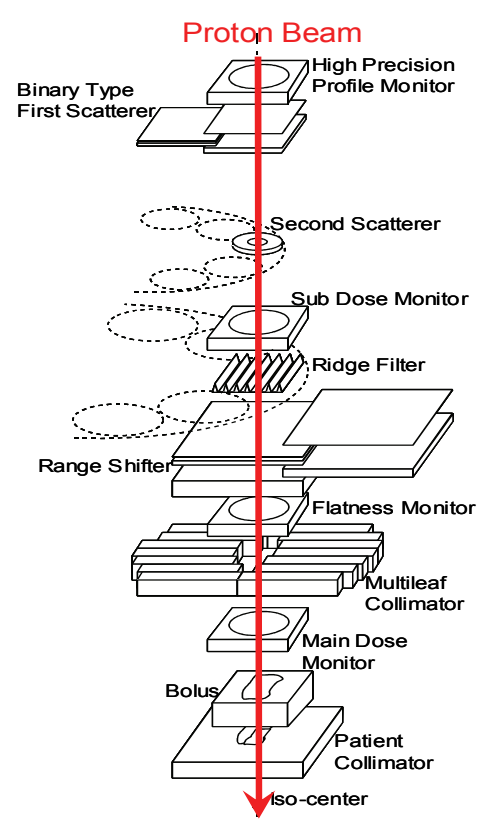

Fig. 3 Outline of proton irradiation devices of PMRC

PMRC facility model. The phantom model was made of $3 \mathrm{~mm}$ thick PMMA and filled with light water. Subsequently, the phantom was irradiated by a $200 \mathrm{MeV}$ proton beam. In the simulation, proton flux, absorbed dose by the proton and their distribution in the phantom were determined by PHITS. Secondary neutrons, photons and incidental doses caused by the secondary radiations in proximity to the phantom were also determined.

In the PHITS estimation, first, to validate the results obtained by PHITS a simulation was carried out using light water as phantom material, thus being able to compare simulation and experimental data reliably. After the validation test, to be able to increase the accuracy of dose deposition with regard to human body tissue, the phantom material is exchanged for a material composition representing human tissue. In our simulation an average normal tissue according to the "Adult ICRU-44(male)" as described in ICRU-46 was applied. ${ }^{15)}$ Table 1 shows the contents for each element applied to the phantom composition for the simulation. The simulation results yield incidental absorbed dose contributions for gamma and proton dose as well as neutron doses for each component.

The absorbed doses by the reaction with neutrons were determined by using the heating number built into ENDF/B-7. Finally, each absorbed dose generated by the reaction between secondary radiation and each element such as hydrogen, nitrogen, oxygen and carbon were determined, respectively. On the other hand, the gamma-ray dose caused by secondary photons was determined by multiplying photon fluxes by the gamma-ray dose conversion factor which is installed in PHITS.

We tried to estimate the equivalent dose based on the calculations of the absorbed doses. However, in the case of neutrons, it is difficult to define a suitable neutron weighting
Table 1 Contents of each element of average normal tissue

\begin{tabular}{lrrrrr}
\hline Tissue & \multicolumn{6}{c}{ Elemental composition $(\mathrm{wt} \%)$} & $\rho\left(\mathrm{kg} / \mathrm{cm}^{3}\right)$ \\
& $\mathrm{H}$ & $\mathrm{C}$ & $\mathrm{N}$ & $\mathrm{O}$ & \\
\hline $\begin{array}{l}\text { Adult ICRU-44 } \\
\text { (male) }\end{array}$ & 10.5 & 25.6 & 2.7 & 60.2 & 1030 \\
\hline
\end{tabular}

factor (or RBE) for the absorbed dose. In the estimation in particular, the absorbed doses were determined for each element; the weighting factor might also change due to change of the neutron energy or spectrum according to the estimation point in the phantom. Therefore, it is impossible to define a suitable weighting factor for each absorbed dose. Nevertheless, in order to evaluate the equivalent dose, we used a uniform weighting factor for each neutron absorbed dose set to 10 . The value is the same as that for the weighting factor applied to the equivalent dose estimation by Clasie et al. $^{12)}$

\section{Results and Discussions}

\section{Estimation Results for the Condition of Phantom Ex- periment}

First, the estimations with PHITS were validated by comparing between calculated values for the proton dose and the measured values obtained from a phantom experiment. Uncertainties of the Monte-Carlo calculations for protons were within 5\%. Uncertainties for neutrons and photons were each within $15 \%$. The calculation value of the proton dose is in good agreement with the measured value within the statistical errors $(5 \%)$. For thermal neutrons $(<0.5 \mathrm{eV})$, the calculated flux values were compared with experimental values gained from the activity of the gold foils, the agreement between the measurement and calculation is within $20 \%$. The calculation value of the gamma-ray dose was also comparable to the experimental value of the CR-39 measurement and within statistical error limits.

On the basis of the results, several calculation values were normalized by the proton dose value at peak point of the dose distribution. Figures 4(a) and (b) show two dimensional distributions in the phantom for calculated neutron flux (a) and photon flux (b) per 1 Gy proton dose. Figure 5 shows profiles of the calculated secondary neutron flux per 1 Gy in the phantom. In this case the profiles are estimates of the flux par 1 Gy proton dose for each cylindrical region in the phantom from beam central axis at intervals of $3 \mathrm{~cm}$, respectively. From the estimation results for the secondary neutrons and photons, the maximum neutron flux per $1 \mathrm{~Gy}$ proton dose was approximately $8 \times 10^{7}\left(\mathrm{n} / \mathrm{cm}^{2}\right) / \mathrm{Gy}$. Among them, the fast neutron flux $(>10 \mathrm{keV})$ and the thermal neutron flux $(<0.5 \mathrm{eV})$ were about $5 \times 10^{7}\left(\mathrm{n} / \mathrm{cm}^{2}\right) / \mathrm{Gy}$ and $2 \times 10^{7}$ $\left(\mathrm{n} / \mathrm{cm}^{2}\right) / \mathrm{Gy}$, respectively. For the secondary photons, intensity and distributions approximately match the neutron values. The estimation results demonstrated that fast neutrons are generated in the vicinity of the proton irradiation field. The results further suggest that the fast neutrons are generated in the upstream devices and from hence they are reaching the phantom surface. The results in Fig. 4(a) and 


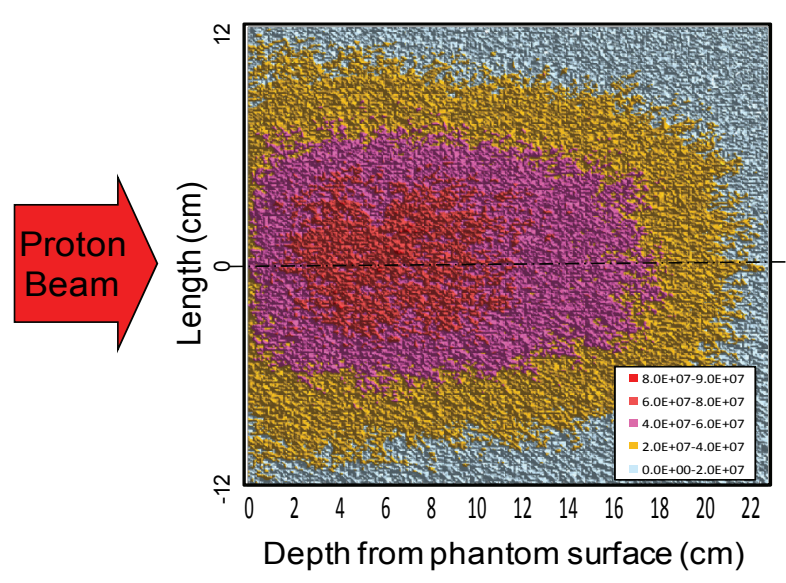

(a) Neutron flux distribution in the phantom

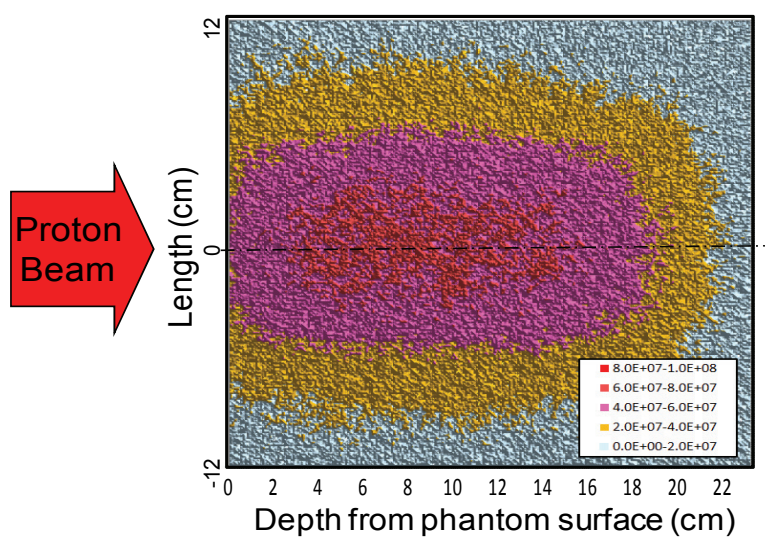

(b) Photon flux distribution in the phantom

Fig. 4 Two dimensional distributions in the phantom for Neutron (a) and Photon (b)

Fig. 5 show that the neutron flux nearby the beam entrance field is higher than the flux at deeper regions due to exposure of the external fast neutrons from the upstream collimator. Both fast neutrons are thermalized in the phantom, the thermal neutrons and the photons are generated and then the secondary radiations spread out ultimately leaving the phantom area.

Next, incidental doses caused by the reaction with secondary radiation and several elements that form human body tissue were estimated. Figure 6 shows profiles of a hydrogen absorbed dose as generated by the reaction between hydrogen and fast neutrons. Figure 7 shows profiles of the gamma-ray dose in the phantom. Maximum value of the hydrogen dose per 1 Gy proton dose was approximately $0.64 \mathrm{mGy} / \mathrm{Gy}$ at the depth of $6.5 \mathrm{~cm}$ on the central axis of the phantom. The maximum value of the gamma-ray dose was approximately $0.45 \mathrm{~m} \mathrm{~Gy} / \mathrm{Gy}$ at the depth of $4-10 \mathrm{~cm}$ along the central axis. The dose at the outer edge region of the phantom was about $0.11 \mathrm{mGy} / \mathrm{Gy}$.

For the estimation of the total incidental equivalent dose, the maximum value of the equivalent dose per $1 \mathrm{~Gy}$ proton therapeutic dose was approximately $8.86 \mathrm{mSv} / \mathrm{Gy}$ at a depth of $6.5 \mathrm{~cm}$ on the central axis in the phantom. The dose at the outer edge region of the phantom was about $2.0 \mathrm{mSv} / \mathrm{Gy}$.

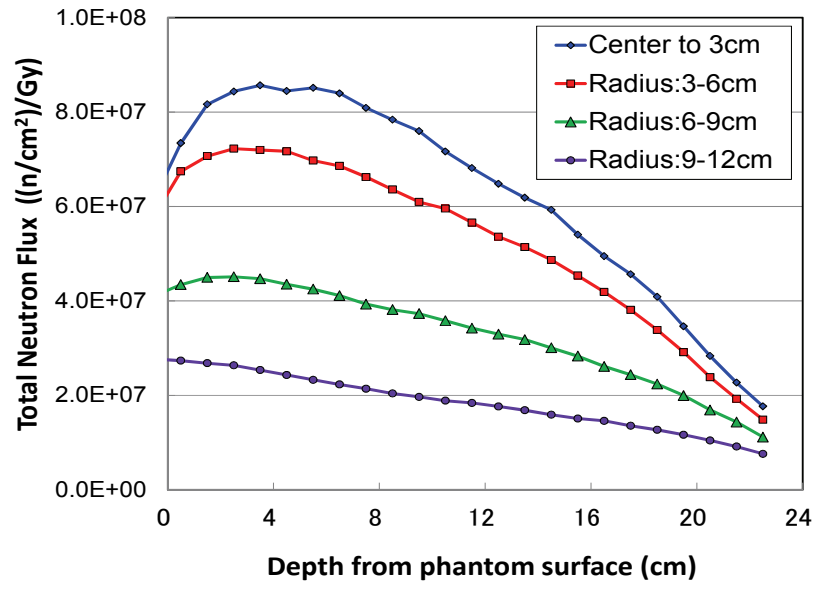

Fig. 5 Profiles of secondary neutron flux in the phantom

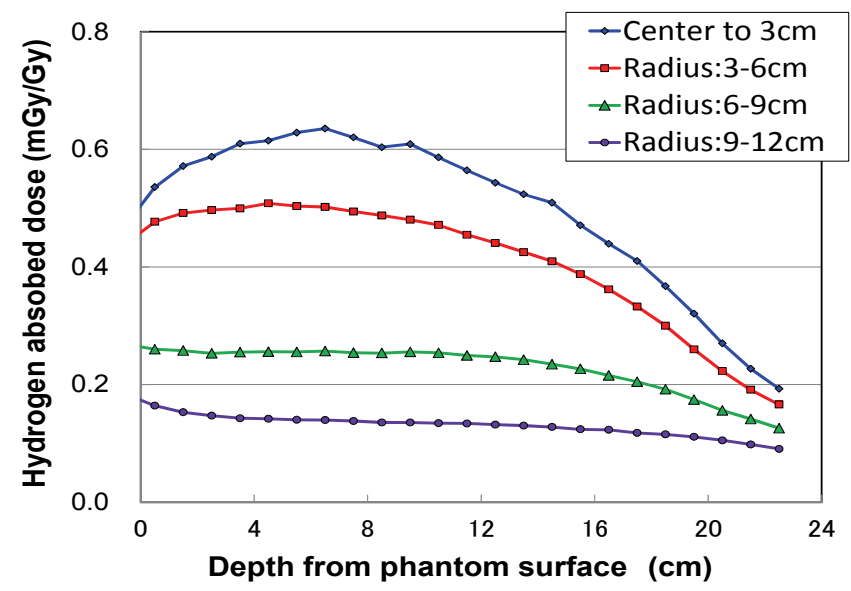

Fig. 6 Profiles of hydrogen absorbed dose in the phantom

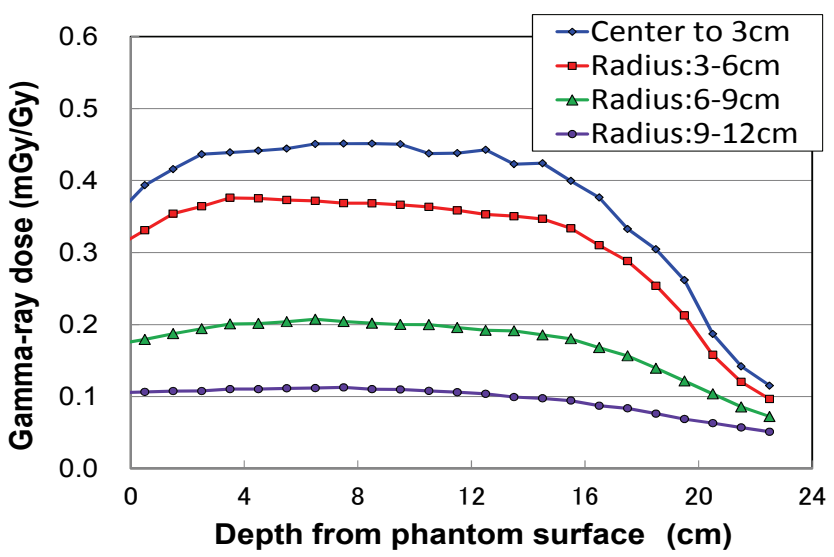

Fig. 7 Profiles of gamma-ray dose in the phantom

The total incidental equivalent dose was composed mainly of hydrogen dose, gamma-ray dose, oxygen dose and nitrogen dose. The estimation results reveal that the hydrogen dose accounted for about $72 \%$ of the total incidental dose. For gamma-ray dose generated by the secondary photons, the amount of the gamma-ray dose at the beam central axis was about $5 \%$ of the total incidental dose. 


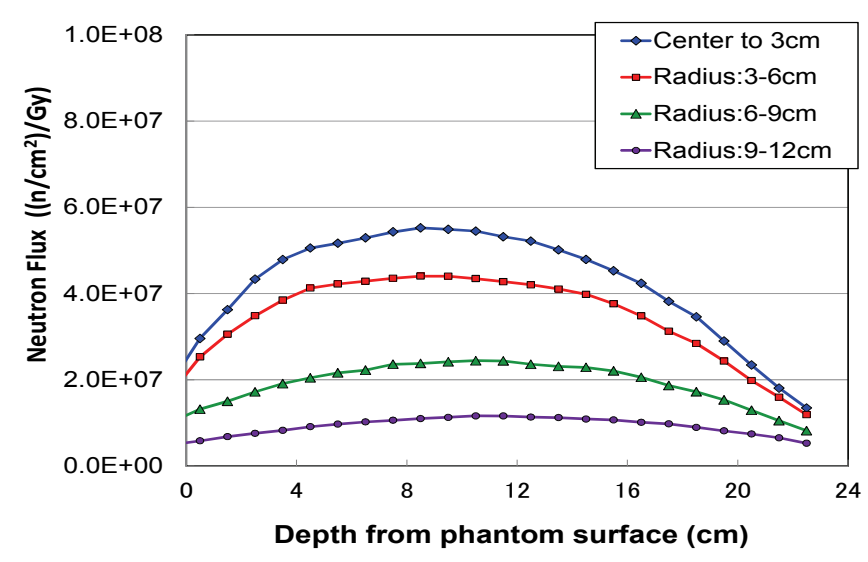

Fig. 8 Profiles of internal neutron flux in the phantom

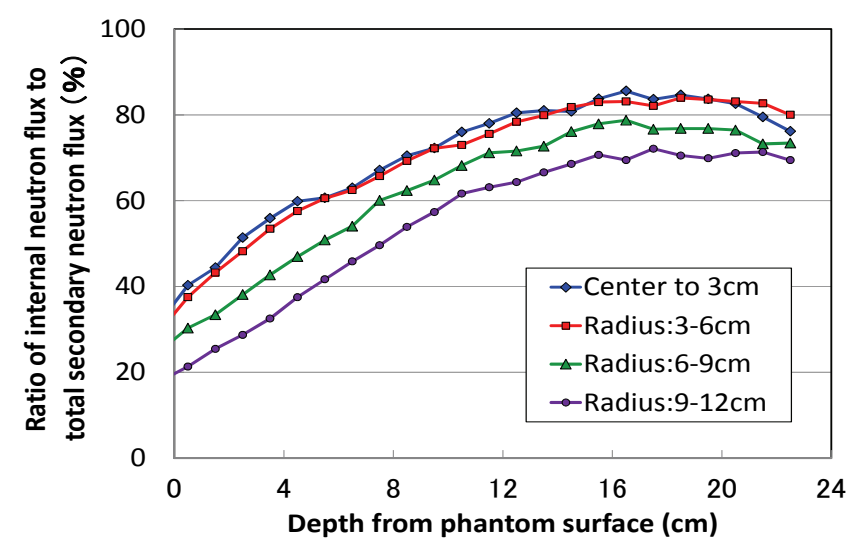

Fig. 9 Ratio of internal neutron flux to total neutron flux

\section{Investigation of Influence of the Internal Secondary Radiations}

Based on the above results, influence of internal secondary radiation and external neutrons from the upstream devices were investigated additionally. With the phantom irradiation model, the external neutrons and photons generated in upstream devices were cut forcibly in the area before the phantom surface, and then the secondary radiation and incidental doses generated in the phantom were determined by PHITS calculation.

Figure 8 shows profiles of internal neutron flux generated in the phantom. And Fig. 9 shows the ratio of internal neutron flux to the total neutron flux shown in Fig. 5. These results demonstrated that percentages of the external neutrons which irradiate the phantom's surface were in the range of $60 \%$ to $80 \%$. For the incidental equivalent dose, the percentage of the dose caused by external neutrons accounted for about $90 \%$ of the total incident dose at the surface field. Therefore, in the proton radiotherapy with passive scattering irradiation method, by shielding and decreasing the external neutrons from the upstream devices, the incidental dose for the beam entrance region can be reduced. Likewise, in radiotherapy utilizing the active spot scanning method, equivalent efficacy might be obtained. However, the results also demonstrate that contributions of the external neutrons to deeper located regions were small, and the internal neutrons generated at the deeper region accounted for a large

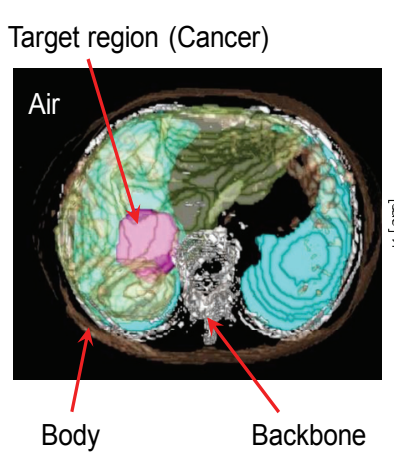

(a) $3 \mathrm{D}$ model

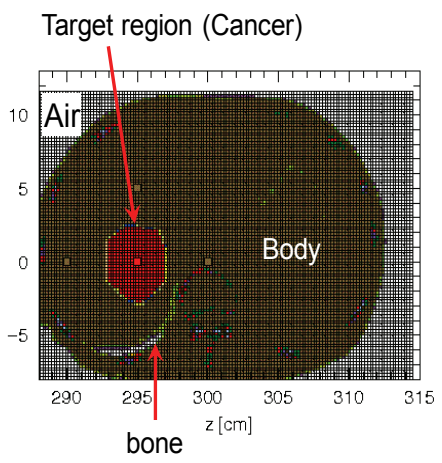

(b) Voxel calculation model
Fig. 10 Three-dimensional model (a) and voxel calculation mode (b) for Proton irradiation simulation with JCDS-FX

amount of the total neutron count. Internal incidental dose around the center at deeper situated regions accounted for about $60 \%$ to $80 \%$ of the total incidental dose. Thus, the results demonstrated that it is impossible to completely eliminate the incidental dose given to a body, even if shielding of the external neutrons or application of the active scanning method are achieved.

\section{Dose Estimation by Using JCDS-FX}

The results of the estimations for the phantom irradiation experiment described above demonstrated that the application of PHITS during treatment planning for proton radiotherapy enables us to perform a dose estimation for not only therapeutic dose of proton radiation but also incidental dose caused by secondary radiation. On the basis of these results, we have begun investigation of Monte-Carlo treatment planning for proton radiotherapy by using JCDS-FX. Dose estimation for proton irradiation of a human body was carried out using JCDS-FX as a basis for initial trials. A three-dimensional human model was created by JCDS-FX using CT images for a trunk of a body, and the target region was set in the liver region on the assumption of proton irradiation against liver cancer. Subsequently, a voxel calculation model consisting of $2 \times 2 \times 2 \mathrm{~mm}^{3}$ voxel cells was created. Figure 10(a) shows the three-dimensional model of the liver cancer, and Fig. 10(b) shows a cross section view of the voxel calculation model of PHITS.

With the voxel calculation model, dose estimation was performed with PHITS, and then distributions of proton dose and secondary radiations around the irradiation field were determined. Figure 11(a) shows the distribution of the therapeutic dose by the proton irradiation around the target region. And the distribution of the secondary neutron flux is shown in Fig. 11(b). The result of the neutron distribution shows that the secondary neutrons spread widely around the body. The results of the preliminary estimation demonstrated that JCDS-FX allows performing dose estimation for a human body treated with proton irradiation and can be implement into the treatment planning for not only BNCT but also proton radiotherapy. To realize practical application of the system to proton radiotherapy, further verifications of the system are being performed. 


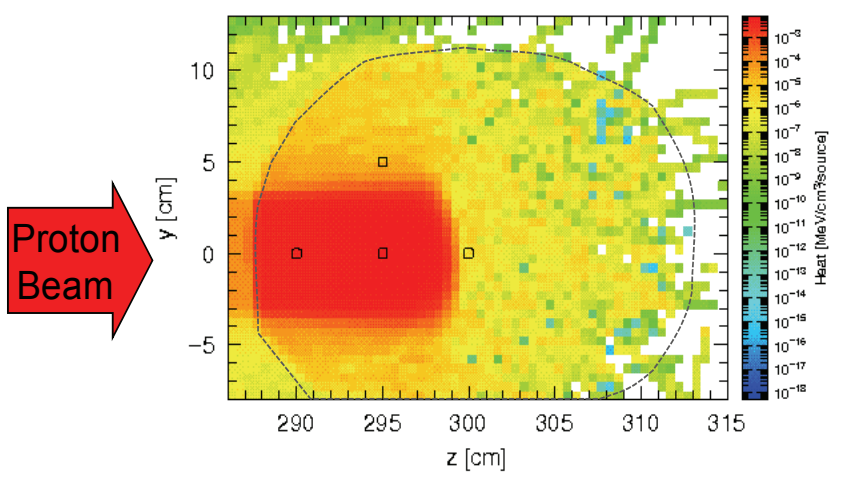

(a) Therapeutic dose distribution

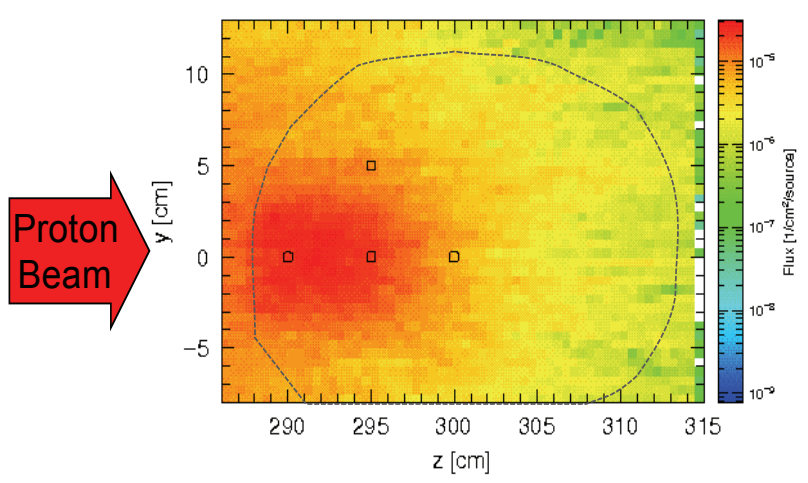

(b) Neutron flux distribution

Fig. 11 Proton dose distribution (a) and neutron flux distribution (b) estimated by JCDS-FX

\section{Conclusion}

In the proton radiotherapy, incidental doses caused by the secondary radiations are given to a patient in addition to therapeutic dose. However, the incidental doses and the secondary radiations do not count toward treatment planning. To improve the dose estimation accuracy of the treatment planning, we intend to apply JCDS-FX to proton radiotherapy.

To estimate the secondary radiations and the incidental doses, a phantom experiment was recreated by using PHITS, and fluxes of the secondary radiation and their distributions in the phantom were determined.

The estimation results demonstrated that the maximum value of the hydrogen dose per 1 Gy proton dose was approximately $0.64 \mathrm{mGy} / \mathrm{Gy}$ at a depth of $6.5 \mathrm{~cm}$ on the central axis of the phantom, while the hydrogen dose accounted for about $72 \%$ of the total incidental dose. The results also indicated that a lot of the external neutrons generated in upstream devices irradiate the surface of the patient's body. However, at regions located more deeply, contributions to dose from external neutrons were small, rather neutrons generated inside the body accounted for a large amount of the total neutron count in the investigated region.

Preliminary estimations with JCDS-FX with regard to a realistic human body model were carried out and the results proved that JCDS-FX can be used to perform treatment planning in the field of proton radiotherapy. To realize practical application of JCDS-FX to proton radiotherapy, further verifications of the system are being performed.

\section{References}

1) M. Umezawa, H. Sakurabata, M. Tadokoro, H. Ootsuka, H. Nishiuchi, K. Saito, K. Matsuda et al., "Beam commissioning of the new proton therapy system for University of Tsukuba," Proc. of Particle Accelerator Conference, 648-650 (2001).

2) R. Kohno, T. Takada, T. Sakae, T. Nohtomi, T. Terunuma, K. Matsumoto, H. Matsuda, "Range-modulated pencil beam algorithm for proton dose calculation," Jpn. J. Appl. Phys., 40, 5187-5189 (2001).

3) R. Kohno, T. Takada, T. Sakae, A. Nohtomi, T. Terunuma, K. Yasuoka, "Experimental evaluation of pencil beam algorithm by measurements of dose distributions of protons traversing an L-shaped phantom," Jpn. J. Appl. Phys., 40, 441-445 (2001).

4) H. Kumada, T. Nakamura, M. Komeda, A. Matsumura "Development of a new multi-modal Monte-Carlo radiotherapy planning system," Appl. Radiat. Isot., 67, 118-121 (2009).

5) H. Kumada, T. Nakamura, M. Komeda, A. Matsumura, "Development of a multi-modal Monte-Carlo radiation treatment planning system combined with PHITS," LASER-DRIVEN RELATIVISTIC PLASMAS APPLIED TO SCIENCE, INDUSTRY AND MEDICINE: 2nd International Symposium, AIP Conference Proceedings, 1153, 377-387 (2009).

6) H. Kumada, K. Yamamoto, A. Matsumura et al., "Development of JCDS, a computational dosimetry system at JAEA for boron neutron capture therapy," J. Phys. Conf. Series, 74, 1-7 (2007).

7) H. Kumada, K. Yamamoto, A. Matsumura, T. Yamamoto, Y. Nakagawa, K. Nakai, T. Kageji, "Verification of the Computational Dosimetry System in JAERI (JCDS) for boron neutron capture therapy," Phys. Med. Biol., 49, 3353-3365 (2004).

8) H. Iwase, K. Niita, T. Nakamura, "Development of General-Purpose Particle and Heavy Ion Transport Monte Carlo Code," J. Nucl. Sci. Technol., 39, 1142-1151 (2002).

9) D. Thomas et al., "Radiation therapy-induced electrical reset of an implantable cardioverter defibrillator device located outside the irradiation field," J. Electrocardiol., 37[1], 73-74 (2004).

10) C. W. Hurkmans et al., "Influence of radiotherapy on the latest generation of implantable cardioverter-defibrillators," Int. J. Radiat. Oncol. Biol. Phys., 63[1], 282-289 (2005).

11) K. Oda, M. Ito, Y. Miyake, J. Yamamoto et al., "Dose-equivalent response CR-39 track detector for personal neutron dosimetry," Nucl. Instr. Meth. Phys. Res., B61, 302-308, (1991).

12) K. Arigane, T. Yamada, D. Nemoto, M. Banba, K. Kawarai, Installation of JRR-2 Neutron Medical Irradiation Facility, JAERI-M 91-39, Japan Atomic Energy Research Institute (JAERI) (1991).

13) B. Clasie, A. Wroe, H. Kooy et al., "Assessment of out-of-field absorbed dose and equivalent dose in proton fields," Med. Phys., 37[1], 311-321, (2010).

14) S. Agostinelli et al., "GEANT4 -A simulation toolkit," Nucl. Instr. Meth. Phys. Res., A506, 250-303 (2003).

15) The International Commission on Radiation Units and Measurements, Photon, Electron, Proton and Neutron Interaction Data for Body Tissue, ICRU-REPORT 46 (1992). 